Department of Mechanics and Automation Engineering, Lviv Polytechnic National University, Ukraine, Lviv, S. Bandery Street 12, E-mail: Volodymyr.I.Hurei@1pnu.ua, ORCID 0000-0002-5117-6802

\title{
WEAR RESISTANCE OF STRENGTHENED LAYERS WITH NANOCRYSTALLINE STRUCTURE IN FRICTION WITH BOUNDARY LUBRICATION
}

Received: March 21, 2020 / Revised: June 15, 2020 / Accepted: July 31, 2020

(c) Gurey V., 2020

\begin{abstract}
Friction treatment refers to surface strengthening (hardening) methods using highly concentrated energy sources. The source of thermal energy occurs in the contact area of the tool-part due to high-speed friction $(60-90 \mathrm{~m} / \mathrm{s})$ of the tool on the treated surface. The heating rate of the metal surface layer is $10^{5}-10^{6} \mathrm{~K} / \mathrm{s}$. After moving the energy source from the contact zone, highspeed cooling of the surface layer of the metal takes place. The cooling rate is $10^{4}-5 \cdot 10^{5} \mathrm{~K} / \mathrm{s}$. Under the action of high-speed heating and cooling of the contact area of the tool-part in the surface layers, a strengthened (hardened) nanocrystalline (white) layer is formed. The formed nanocrystalline surface layer has other physical, mechanical, chemical properties in comparison with the base metal of the part. Studies have shown that in the process of friction treatment of working surfaces of parts made of Steel 40NiCr6 (quench hardening and low-temperature tempering) a strengthened layer with a thickness of 250-320 $\mu \mathrm{m}$ with a hardness of 7.6-9.2 GPa is formed. The grain size of the surface strengthened layer was $20-40 \mathrm{~nm}$ near the treated surface. The formation of the strengthened layer is influenced by the shape of the working surface of the tool. Thus, a strengthened layer of greater thickness and hardness is obtained when machining with a tool with transverse grooves on the working part than with a tool with a smooth working part. Experimental studies in friction with maximum lubrication of pair "Steel 40NiCr6 and Grey Cast Iron GG20" showed that the strengthened nanocrystalline layer significantly increases the performance during sliding friction. Only samples made of steel were strengthened, counter-samples made of gray cast iron were not strengthened. The wear intensity of strengthened pair is 2.2-3.1 times less compared to unstrengthened pair. During the friction of the strengthened pair, the coefficient of friction and the temperature in the sliding zone also decrease. The best results were obtained when studying the friction pair in which the samples were strengthened with a tool with transverse grooves on its working part.
\end{abstract}

Keywords: white layer, wear, wear resistance, nanocrystalline structure, friction treatment, boundary lubrication.

\section{Introduction}

Heat treatment of the entire volume of the part has exhausted its possibilities. To increase the durability of machine parts, it is necessary to improve the properties of the surface layers. This can be made by applying different coatings, as well as by modifying the surface layer. The durability of coatings depends on their adhesion to the base metal of the part. During the modification, its characteristics change, but the crystal lattice remains the same, only its parameters change. One of the methods of surface layer modification is the formation of nanocrystalline structures in it. The surface layer with a nanocrystalline structure is obtained after frictional treatment of the working surfaces of machine parts. The obtained reinforced layer has increased hardness, dislocation density, the amount of residual austenite and other 


\section{Volodymyr Gurey}

parameters. In this work, studies of wear resistance during friction without lubrication and with maximum lubrication of strengthened pairs.

\section{Experimental Procedure}

The service properties of parts and assemblies (units) significantly depend on the quality of the contact surfaces and the surface layer, which is determined by the geometrical, physical and mechanical characteristics and the relative position of micro-irregularities on the contact surfaces. All these parameters depend on the technology of machining parts and assembly of products.

Significantly increase the durability, speed, load of components and machines we can by changing the technological processes which improve the service properties of the surface layer of the machine parts. Using new and modern methods of surface treatment is determined not only by the conditions of high production, but also by creation the surfaces with optimal and high load capacity, which is especially important when this contact surfaces are working with friction and wearing under the influence of fatigue loads. This gives an opportunity to significantly improve the operating parameters and characteristics of manufactured products and machines [1].

The reliability of the machines is directly related to the quality of the surface layer of parts, which is characterized by geometrical, physical and mechanical parameters. The parts are in contact with each other and with the environment during the operation. The quality of the surface layer depends on the service properties - resistance to fatigue failure, wear resistance, corrosion resistance and others. The connection between the quality characteristics of the surface layer and the service properties of the parts indicates (shows) that the optimal surface should be sufficiently hard, have residual compressive stresses, finely dispersed structure, smoothed shape of microroughness with a large area of the bearing (supporting) surface [2].

The operational characteristics of machine parts and mechanisms often depend not so much on the quality of the metal of the whole cross-section as on the physicochemical properties of the surface layer and the state of the surface of products that are formed at the final stages of manufacturing of parts [1].

Currently, methods of surface treatment and hardening (strengthening) by highly concentrated energy sources are used. These are laser, plasma, friction and other treatments [3], [4]. During these methods, high-intensity concentrated energy flows act on the local volumes of the surface layer of the metal at high speeds, and when the energy source is removed, they are rapidly cooled. The surface layers of parts undergo structural and phase transformations with the formation of reinforced layers, which have specific physical and mechanical, electrochemical, corrosion and service characteristics, which significantly effect on the performance of machine parts and units during operation [5], [6].

\section{Methodology}

Friction treatment refers to surface hardening (strengthening) technologies with highly concentrated energy flows. Highly concentrated energy flow is formed in the contact zone due high speed $(60-90 \mathrm{~m} / \mathrm{s})$ friction of the metal tool-disk on the workpiece. At the same time there is an intensive shear deformation. A large amount of technological medium was fed into the processing (strengthening) zone [7].

Frictional treatment of flat surfaces was performed on an upgraded grinding machine. Since the maximum speed during grinding does not exceed $V_{\text {tool }}=36 \mathrm{~m} / \mathrm{s}$, but for the implementation of frictional treatment requires a speed less than $V_{\text {tool }}=60 \mathrm{~m} / \mathrm{s}$, the main drive unit of the machine was modernized. Instead of an abrasive disk, a metal tool-disk made of stainless steel was mounted. The diameter of the tool-disk is $200-210 \mathrm{~mm}$, the width of the working part $-8-10 \mathrm{~mm}$.

To reduce the beating and vibration of the spindle assembly, the tool disk was mounted on the cone of the machine spindle. Before mounting the tool-disk on the machine, its static balancing was performed. The roughness of the working surface of the disk is not more than $R a=0.32 \mu \mathrm{m}$, radial beating - not more than $0.03 \mathrm{~mm}$.

The tool disk was made with a smooth working part and with cut transverse grooves on the working part to increase the shear deformation of the surface layer of metal and provide pulsed friction treatment. 


\section{Wear Resistance of Strengthened Layers with Nanocrystalline Structure in Friction with ...}

The width of the grooves was chosen so as to ensure complete escaping (runaway) from the contact of the tool with the part.

As a technological medium in the process of friction treatment used mineral oil with additives containing polymers. To fed the process medium into the processing (strengthening) zone a coolant system of the machine was used.

The technological medium, which is fed into the processing (strengthening) zone, eliminates the setting of the tool-disk and the workpiece and helps to improve the roughness of the reinforced surface and the quality of the part. In the process of frictional treatment, the mineral oil with additives containing polymers decomposes into atomic carbon and undergoes its mass transfer (diffusion) into the surface layers of the workpiece, which contributes to the formation of high-quality reinforced layers.

Studies of wear resistance were carried out by friction with boundary lubrication of pairs "Steel 40NiCr6 (DIN) - Grey Cast Iron GG20 (DIN)" according to the scheme "Ring-Ring" on a universal friction equipment (UMT-1, USSR). The samples used for research are shown in Fig. 1. The sliding speed was varied from $0.2 \mathrm{~m} / \mathrm{s}$ to $4 \mathrm{~m} / \mathrm{s}$. The load on the samples was adjusted in the range from $0.1 \mathrm{MPa}$ to 1.5 $\mathrm{MPa}$. The stationary (fixed) sample was connected to a differential power meter sensor and the signal from which was fed to recording device and recorded the friction of the pair. Thermocouples were fixed on the stationary sample (diameter $-0.2 \mathrm{~mm}$ ). Signals from thermocouples were fed to the recording device. The integrated temperature under the friction zone at a depth of $0.3-0.5 \mathrm{~mm}$ below the friction surface was recorded. The friction pair test time at one stage was 8 hours.
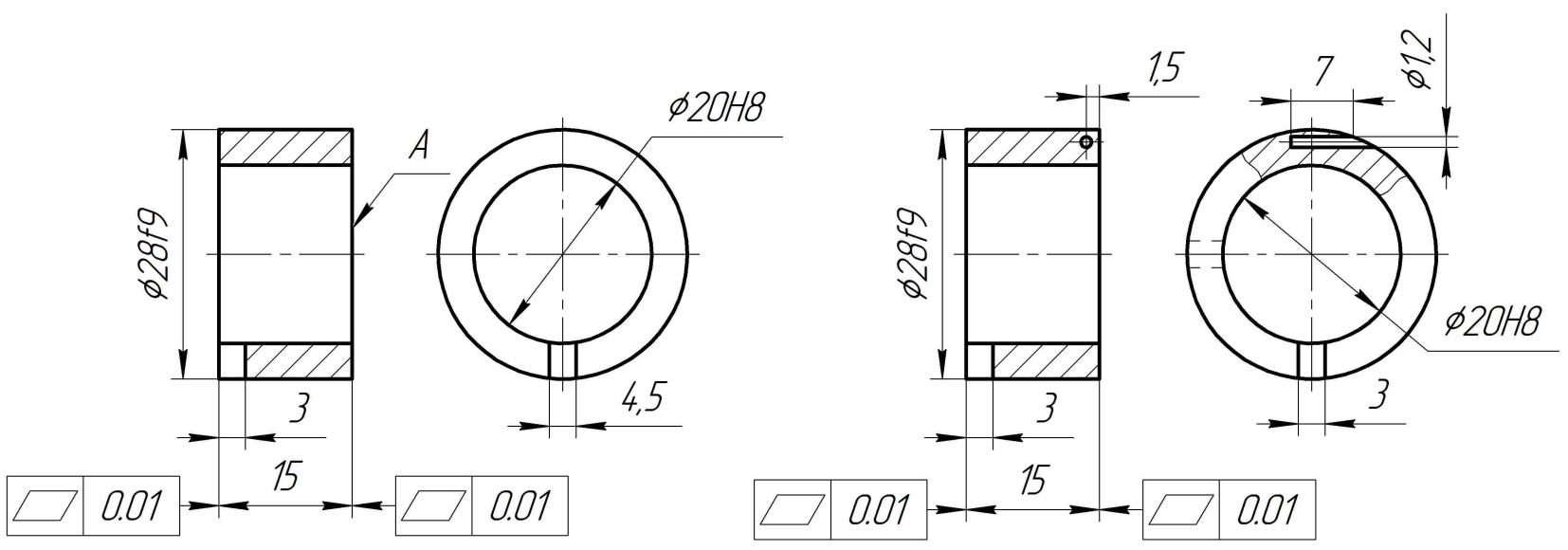

Fig. 1. Sketch of the sample and counter-sample for studies the wear during sliding friction according to the scheme "Ring-Ring"

During the study of wear resistance with boundary lubrication in the area of friction was fed mineral oil 15-20 drops per minute using a special chamber.

Before the start of the experiments, the friction pair was run-in to stabilize the friction moment and adjoin the conjugate surfaces, which are characterized by friction traces on the area of at least $90 \%$ of the working friction surface of each pair's sample.

Samples (movable) of the studied friction pairs were made of Steel 40NiCr6 (DIN) (quench hardening and low-temperature tempering, HRC 48-51), counter-samples (stationary) were made of Grey Cast Iron GG20 (DIN). Working surfaces of movable samples of friction pairs were strengthened by friction treatment, stationary samples were only ground (unstrengthened). When strengthening both bodies of the friction pair, the effect of increasing wear resistance is missing. Unchanged friction pair was used for comparison. The roughness of the working surfaces after friction treatment was $R a=0.25-0.50 \mu \mathrm{m}$, after grinding $-R a=0.50-0.63 \mu \mathrm{m}$.

The criterion for the amount of wear was taken as the loss of mass of the samples after each stage of friction, which was determined by weighing on an analytical balance with an accuracy of $\pm 0.2 \mathrm{mg}$. After that, the intensity of wear of the friction pair elements was determined. 


\section{Volodymyr Gurey}

\section{Results and Discussion}

Metallographic analysis showed that after friction treatment of Steel $40 \mathrm{NiCr} 6$ (DIN) samples (specimens) (quench hardening and low-temperature tempering) using the tool with a smooth working part formed a reinforced surface layer with a nanocrystalline structure (white layer) and a thickness is 200$220 \mu \mathrm{m}$ (Fig.2). The microhardness of the reinforced layer is 7.2-7.8 GPa and a hardness of the base metal is $4.7-5.2 \mathrm{GPa}$ (Fig. 3).

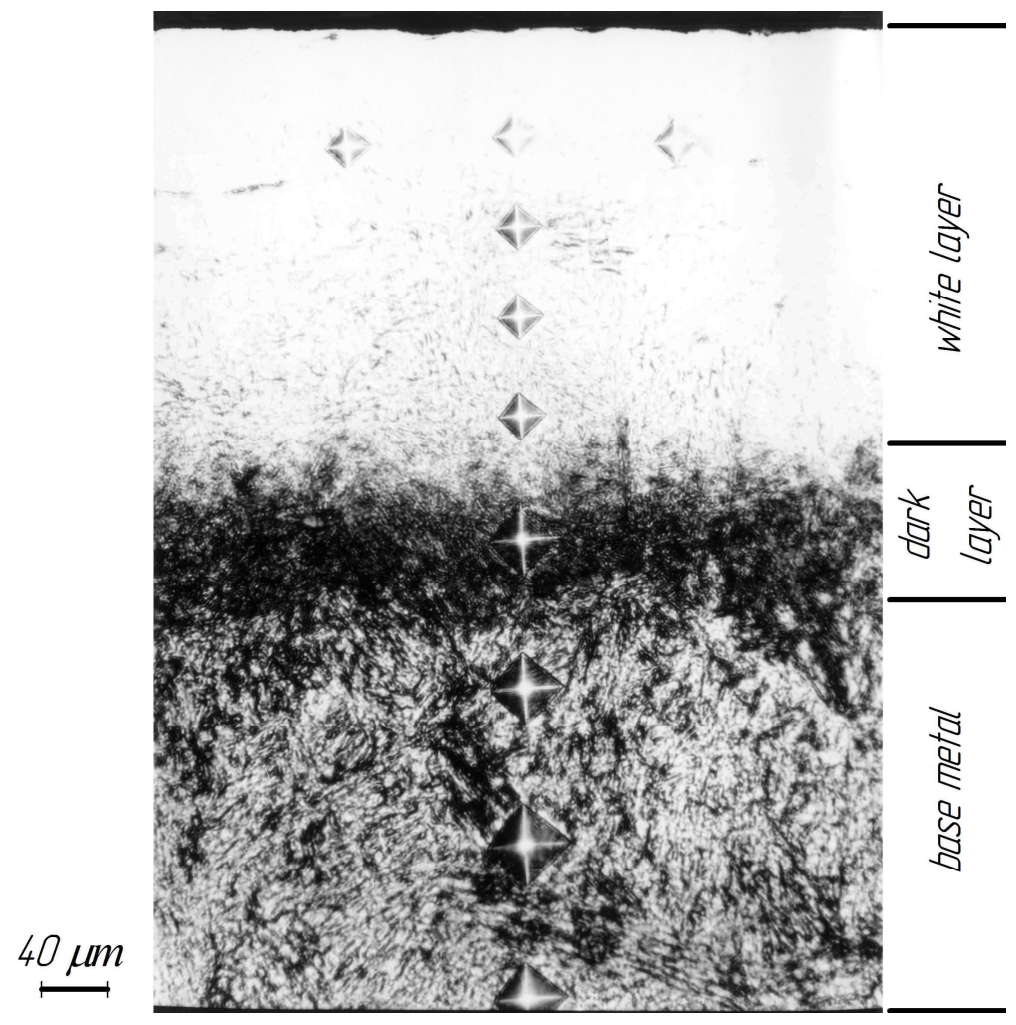

Fig. 2. Microstructure of the reinforced (strengthened) layer after frictional treatment of Steel 40NiCr6 (DIN) (quench hardening and low-temperature tempering) (the tool with smooth surface of a working part)

During the process of frictional treatment using the tool with a smooth working part, there are only shear deformations in the area of contact of the tool-part which formed under the action of high-speed friction of the tool on the processed surface. At the moment when the groove is above the contact area of the tool-part due to elastic deformation the surface layers return to their original position using the tool with transverse grooves on the working part. At the time of contact of the next continuous smooth section of the tool is the shock load of the surface layer of the part in the contact area of the tool-part. In the surface layer of the part, in addition to shear deformation due to high-speed friction, high-speed shock deformation is added.

The thickness of the reinforced layer increased to 300-320 $\mu \mathrm{m}$, after machining with a tool which has transverse grooves. The hardness also increased to 8.6-8.8 GPa. In the process of frictional treatment, when using the tool with grooves on the working part there is an intense shear alternating deformation of the surface layer of metal, which extends to a greater depth than when using the tool with a smooth working part.

Under the white layer obtained on the samples after quench hardening and low-temperature tempering, there is a dark layer with reduced hardness than the base metal. In this zone, the metal is heated to temperatures below the phase transition point. This heating is not enough to form a reinforced layer, but enough to release the pre-hardened (by quench hardening) metal structure. Also, during the process of friction treatment there is a change in the chemical composition of the reinforced layer. Mass transfer (diffusion) takes place both from the technological environment and from the layers under the reinforced layer [8]. No dark layer is observed during friction treatment of the samples after quench hardening and high-temperature tempering and after normalization. 
Wear Resistance of Strengthened Layers with Nanocrystalline Structure in Friction with ...

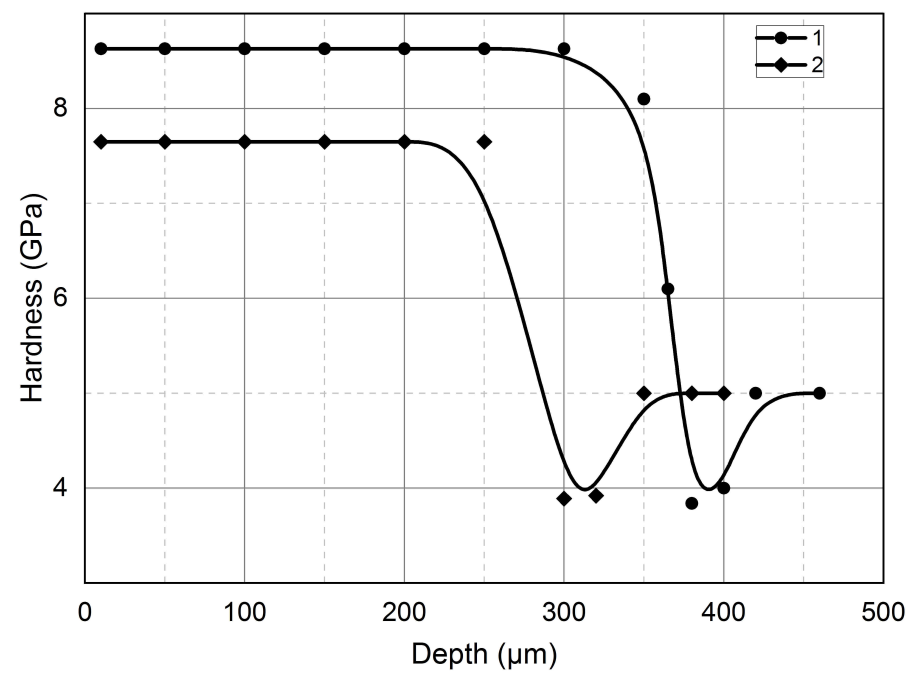

Fig. 3. Microhardness of the reinforced (strengthened) layer after frictional treatment of Steel 40NiCr6 (DIN) (quench hardening and low-temperature tempering) the tool with a different form of a working part: 1 - with cross grooves; 2 - smooth surface

X-ray analysis showed that the grain size of the surface reinforced layer was about 20-40 nm near the surface. With increasing depth of the reinforced layer, the grain size increases and smoothly transitions to the structure of the base metal. The structure of the obtained reinforced surface layer after friction treatment refers to nanocrystalline.

Wear of machine parts is characterized by the destruction of the surface layer. The contact interaction of the surfaces of the parts determines the actual contact area, contact stiffness, the magnitude of local stresses, the conditions of formation of the oil film, the adhesion of the coatings and others. Wear resistance and contact strength mainly depend on such surface quality characteristics as its microhardness and the magnitude and sign of residual stresses [9].

The working surfaces of the movable samples, made of Steel 40NiCr6 (DIN), were strengthened by friction treatment with a disk tool with a smooth working surface and a tool with transverse grooves on the working part. The working surfaces of the counter-samples, made of Grey Cast Iron GG20 (DIN), were not reinforced.

Studies have shown that frictional treatment significantly increases the wear resistance during friction with boundary lubrication (Fig. 4). At low speeds sliding $V=0.2 \mathrm{~m} / \mathrm{s}$ the wear resistance increases by $2.2-2.5$ times. The effect of frictional treatment increases when the sliding speed increases. The largest increase of wear resistance is observed at a sliding speed $V=1.5 \mathrm{~m} / \mathrm{s}$ and is $2.9-3.2$ times. The wear resistance of the samples after friction treatment by a tool with transverse grooves is slightly higher in comparison with the samples strengthened by a tool with a smooth working part. The nature of wear of non-reinforced counter-samples, which worked in pair with reinforced samples, is the same as that as reinforced ones.

When increase the sliding speed more than $1,5 \mathrm{~m} / \mathrm{s}$ all friction pairs become unserviceable. In the latter case, the main role is played the temperature in the friction zone (Fig. 5). In the process of friction of strengthened samples, the temperature in the contact zone is lower as compared with unstrengthened pair. The boundary film of lubricant is destroyed and begins to setting, further work of the friction pair becomes impossible when in the friction zone reaches of a temperature about $250^{\circ} \mathrm{C}$. In the process of frictional treatment using a tool with a smooth working part significantly reduces the friction coefficient over the entire range of the studied friction speeds. But when using the tool with transverse grooves on the working part further reduces the friction coefficient (Fig. 6).

All lubricants have the ability to adsorb on the metal surface. Surface wetting depends on the nature of the lubricating medium, surface energy and roughness of the friction surfaces. Lubricant molecules are oriented perpendicular to the friction surface, which can be represented as pile. The lubricant in the boundary layer is anisotropic, in the tangential direction the molecular layers are easily bend, at their large thickness they slide one after another. Normally to the friction surface, they have a high compressive strength. 


\section{Volodymyr Gurey}
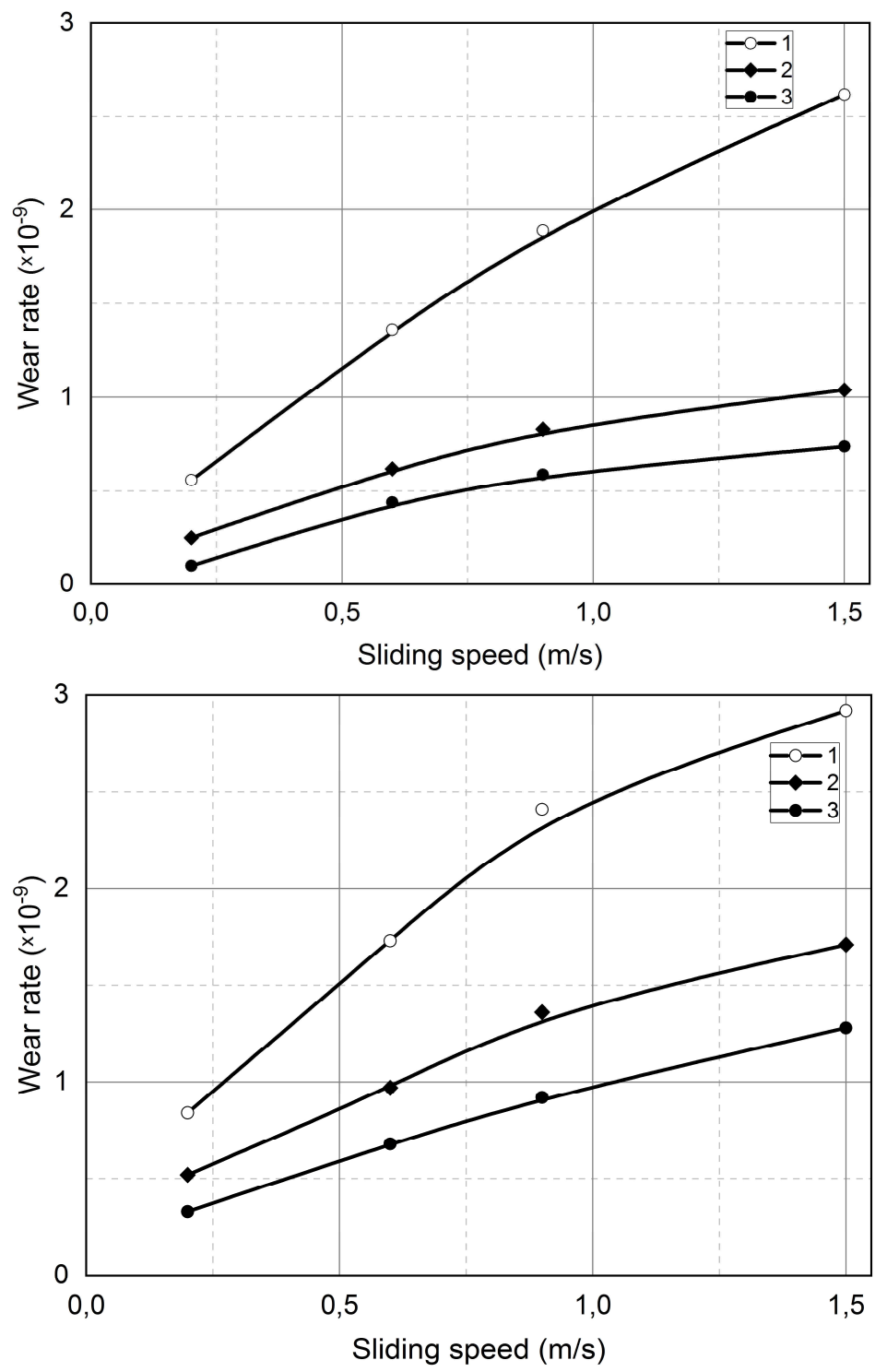

Fig. 4. Wear intensity of sample (a) and counter-sample (b) dependence of sliding speed due friction with boundary lubrication of pair "Steel 40NiCr6 (DIN) (quench hardening and low-temperature tempering) -

Steel 40NiCr6 (DIN) (quench hardening and low-temperature tempering)" after friction treatment $(\mathrm{P}=0.8 \mathrm{MPa})$ : 1 - unstrengthened; 2 - friction treatment, the tool with a smooth working part; 3 - friction treatment, the tool with cross grooves

During friction with boundary lubrication, secondary structures are formed on the friction surfaces, similarly to friction without lubrication. They consist mainly of iron oxides and alloying elements, as well as compounds of active lubricant elements with metal atoms. The elements that form compound with the base material, they can diffuse from both the environment and the volume of the metal.

Lubricants play the role of a screen that reduces the flow of oxygen into the friction zone, as their oxygen content is much lower than in air. The value of oxygen is sufficient for tribochemical reactions in the process of friction with a relatively low level of structural and thermal activation. The level of structural-thermal activation increases sharply, the oxygen content adsorbed on the juvenile surfaces may not be sufficient for the formation of high-quality secondary structures when friction takes place in severe conditions. Additives containing chlorine, sulfur, phosphorus and other elements are added to the oil to 
improve the quality and accelerate the formation of secondary structures. During the process of friction, the ascending diffusion of the reduced elements from under the surface layer into the friction zone can also take place.

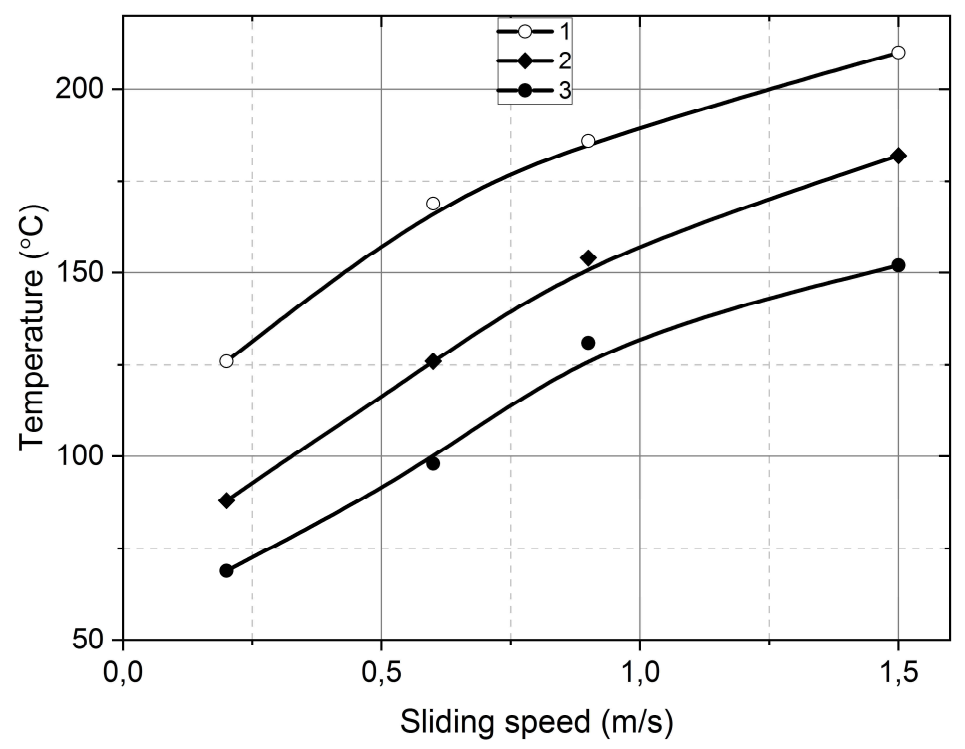

Fig. 5. The temperature in the contact zone dependence of the sliding speed during friction with boundary lubrication of pair "Steel 40NiCr6 (DIN) (quench hardening and lowtemperature tempering) - Steel 40NiCr6 (DIN)

(quench hardening and low-temperature tempering)" after friction treatment $(\mathrm{P}=1$ $\mathrm{MPa}$ ): 1 - unstrengthened samples; 2 - friction treatment, the tool with a smooth working part;

3 - friction treatment, the tool with cross grooves

The wear intensity of both strengthened and unstrengthened pairs is relatively small at friction velocities. The wear rate of strengthened pair is almost 2 times less than unstrengthened. When the speed of friction increases, the wear intensity of the pairs also increases, and the wear intensity of the unstrengthened pairs increases especially strongly. The value of oxygen that is adsorbed on the friction surfaces is sufficient for the formation of high-quality secondary structures when friction of unstrengthened pair at low speeds. The oxygen content becomes insufficient for the formation of high-quality secondary structures when increasing friction modes.

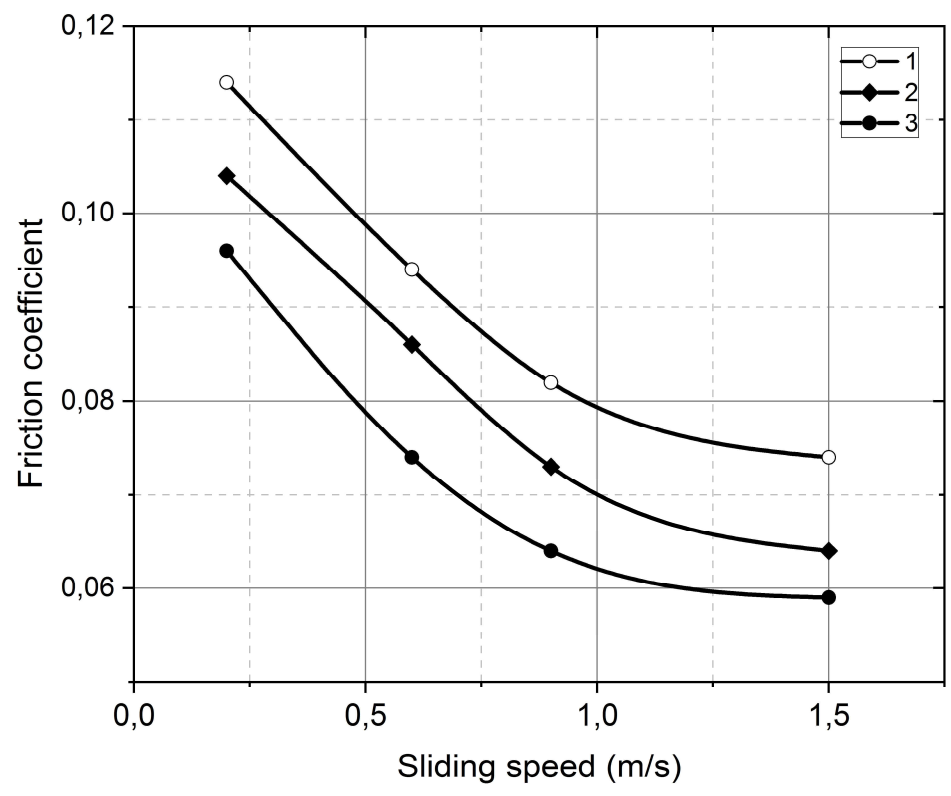

Fig. 6. The friction coefficient dependence of the sliding speed during friction with boundary lubrication of pair "Steel 40NiCr6 (DIN) - Steel 40NiCr6 (DIN) (quench hardening and low-temperature tempering)" after friction treatment $(\mathrm{P}=0.8 \mathrm{MPa})$ : 1 - unstrengthened; 2 - friction treatment, the tool with a smooth working part; 3 - friction treatment, the tool with cross grooves 


\section{Volodymyr Gurey}

Elastic and plastic deformation of the metal occur at the surfaces of contact which coated with a boundary film of lubricant. The sliding resistance consists of the shear resistance of the boundary layer and the resistance to scratching the surfaces by compressed volumes. On the contact surfaces formed a significant plastic deformation and high local temperature occur the destruction of the lubricating film. The adsorption proceeds at a high rate due to the mobility of lubricant molecules on the friction surfaces and which allows to restore the continuity of the boundary film.

The serviceability of friction pairs at the limit friction is limited by the temperature that occurs in the friction zone. On juvenile surfaces occur a setting with pulling out (wresting) of metal and pair becomes unserviceable.

\section{Conclusions}

A strengthened (hardened) nanocrystalline layer is formed in a surface layer in the course of friction treatment of steel components, the layer thickness of which is $250-320 \mu \mathrm{m}$, and the microhardness is 7.68.6 GPa. Grain size of the strengthened (hardened) surface layer was $20-40 \mathrm{~nm}$ near the treated surface.

The strengthened layer significantly increases performance of the pair "steel-grey cast iron" during sliding dry friction.

The wear intensity of strengthened pair is 2.2-3.1 times less compared to unstrengthened pair.

Only one component of the friction pair was strengthened. After the strengthening of both friction pairs, the effect of the increase in wear resistance is leveled off.

\section{References}

[1] V. D. Evdokimov, L. P. Klimenko, and A. N. Evdokimova, Tekhnologiya uprochneniya mashinostroitelnykh materialov [Technology of hardening of machine building materials]. Odesa-Mykolaiv, Ukraine: NSGU Publ., 2005. [in Russian].

[2] K. A. Yushchenko, Yu. S. Borisov, V. D. Kuznetsov, and V. M. Korzh, Inzheneriya poverkhni [Surface Engineering]. Kyiv, Ukraine: Naukova dumka Publ., 2007. [in Ukrainian].

[3] S. Akcan, S. Shah, S. P. Moylan, P. N. Chhabra, S. Chandrasekar, and H. T. Y. Yang, "Formation of white layers in steels by machining and their characteristics", Metallurgical and materials transaction A, vol. 33, pp. 1245$1254,2002$.

[4] Xiangming Huang, Zhixiong Zhou, Yinghui Ren, Cong Mao, and Wei Li, "Experimental research material characteristics effect on white layers formation in grinding of hardened steel", The International Journal of Advanced Manufacturing Technology, vol. 66, pp. 1555-1561, 2013.

[5] C. Cappellini, A. Attanasio, G. Rotella, and D. Umbrello, "Formation of white and dark layers in hard cutting: influence of tool wear", International Journal of Material Forming, vol. 1, no. 3, pp. 455-458, 2010.

[6] H. Gleiter, "Nanostructured materials: basic concepts and microstructure", Acta Materialia, vol. 48, no. 1, pp. $1-29,2000$.

[7] I. Hurey, V. Gurey, P. Dmyterko, and R. Babiarz, "The research in to components of friction force tool part during friction hardening of plate steel faces", Advances in manufacturing science and technology, vol. 3, pp. 56-64, 2014.

[8] I. V. Hurei, T. A. Hurei, and V. V. Tykhonovych, "Redistribution of chemical elements in the process of pulse strengthening", Materials Science, vol. 35, pp. 146-148, 1999.

[9] I. Hutchings, and P. Shipway, Tribology. Friction and wear of engineering materials. Oxford, UK: Butterworth-Heinemann Publ., 2017. 